\title{
Potencial uso de Resíduos de Lodo de Efluente de Indústria de Papel e Embalagens como Fertilizante Agrícola
}

\author{
William D. Miranda, Geymme S. C. Soares, Adriano M. Moura \& \\ Vanessa S. Leal
}

A grande demanda nos processos industriais tem levado à geração de uma quantidade significativa de resíduos sólidos industriais. Tais resíduos apresentam como suas características um grande impacto ambiental. A gestão adequada destes resíduos irá trazer benefícios ao meio ambiental e à saúde humana. $\mathrm{O}$ estudo de caso avaliado possibilita uma aplicação do lodo gerado na indústria de papel, na forma de complemento de fertilizante agrícola, apresentando teores relevantes do macronutriente nitrogênio $(\mathrm{N})$ na ordem de $75 \mathrm{mg} / \mathrm{kg}$ e fósforo (P) 2,0 mg/kg.

Palavras-chave: lodo-fertilizante-residuo.

The great demand in industrial processes has led to the generation of a significant amount of industrial wastes. Such residues present as its features a large environmental impact. Proper management of these wastes will benefit the environmental and human health. The reported case study provides an application sludge generated in paper industry, as agricultural fertilizer complement, showing relevant contents of the macronutrient nitrogen $(\mathrm{N})$ in the order of $75 \mathrm{mg} / \mathrm{kg}$ and phosphorus $(\mathrm{P}) 2.0 \mathrm{mg} / \mathrm{kg}$.

Keywords: sludge-fertilizer - residue. 


\section{Introdução}

Resíduos são considerados tudo aquilo que não é aproveitado nas atividades humanas, resultantes de residências, comércios, redes pública, indústrias e outros. Os resíduos industriais são uns dos maiores responsáveis pelos danos ambientais, pois, dependendo do tipo de industrialização, podem conter produtos químicos, resíduos sólidos, cinzas, gases, plásticos, lodos, óleos, papel, madeira, fibras, borracha, metal, cerâmicas, vidros e diversos outros, podendo ser danosos o meio ambiente (VALOIS, 2013). Devido a isso, as empresas devem possuir um plano de gerenciamento desses resíduos, o que já vem acontecendo na atualidade, devido às exigências das legislações ambientais e da aprovação da Política Nacional de Resíduos Sólidos (PNRS), que determina que todas as indústrias deverão realizar diagnósticos da situação atual em relação à geração e gestão de resíduos, elaborar um Plano de Gerenciamento de Resíduos Sólidos (PGRS) e apresentar aos órgãos competentes do Estado onde está localizada (ALMEIDA et al, 2014).

Os fertilizantes são compostos orgânicos ou inorgânicos utilizados para repor os nutrientes do solo que são essenciais ao desenvolvimento vegetal. O lodo em questão é um fertilizante orgânico vegetal, podendo ser obtido através do processo de compostagem. Este fertilizante fornece nutrientes ao solo e contribui para o melhor desenvolvimento das plantações, sendo um meio sustentável, já que é produzido a partir da reciclagem de resíduos orgânicos (SILVA, 2008).

No processo industrial em questão, o processo de formação do lodo se dá pela estação de tratamento de efluente da indústria. Os processos de tratamento na empresa são classificados em físico-químicos e biológicos. O tratamento primário é constituído por sistemas físico-químicos, gerando, a partir disso, o lodo que é centrifugado, armazenado e disposto no aterro sanitário do município. No processo de tratamento do efluente, são geradas cerca de 350 toneladas de lodo (Figura 1) por mês, na proporção de $60 \%$, pois este é centrifugado restando, portanto, 140 toneladas de lodo seco.

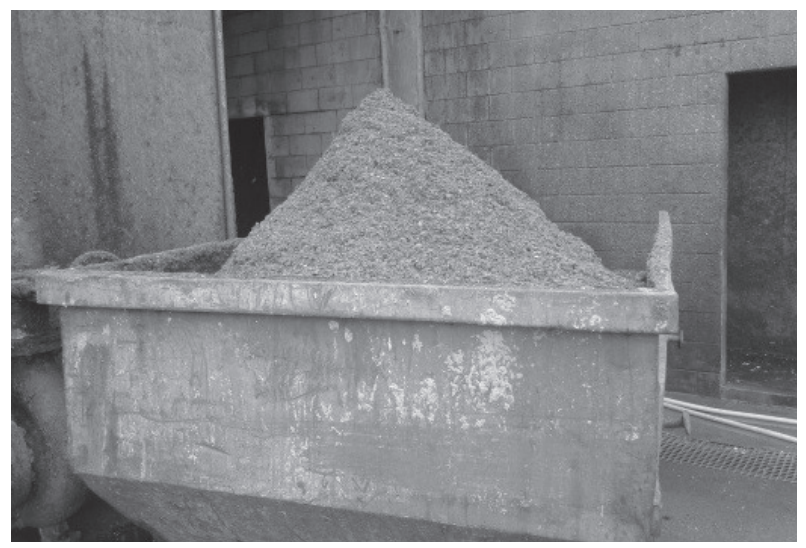

Figura 1. Lodo centrifugado.

Segundo o Decreto 86.955/82 do Ministério da Agricultura, Pecuária e Abastecimento, fertilizantes são "substâncias minerais ou orgânicas, naturais ou sintéticas, fornecedoras de um ou mais nutrientes das plantas". Eles têm a função de repor ao solo os elementos retirados em cada colheita, com a finalidade de manter ou mesmo ampliar o seu potencial produtivo, elevando a produtividade em certas culturas. Os principais nutrientes químicos, encontrados nos fertilizantes, são classificados em duas categorias, são elas: macronutrientes (carbono, hidrogênio, oxigênio, nitrogênio, fósforo, potássio, cálcio, magnésio e enxofre) e micronutrientes (boro, cloro, cobre, ferro, manganês, molibdênio, zinco, sódio, silício e cobalto).

Este artigo tem a função de avaliar os teores percentuais dos macronutrientes de maior deficiência nos solos, nitrogênio $(\mathrm{N})$, fósforo $(\mathrm{P})$ e potássio $(\mathrm{K})$ - NPK. Segundo Fernandes \& Dias (2006), o nitrogênio, como importante componente das proteínas e da clorofila, frequentemente, é fator primordial no aumento da produtividade agrícola. O fósforo é responsável pelos processos vitais das plantas, pelo armazenamento e utilização de energia, promove o crescimento das raízes e a melhora da qualidade dos grãos, além de acelerar o amadurecimento dos frutos. O potássio é responsável pelo equilíbrio de cargas no interior das células vegetais, inclusive pelo controle da hidratação e das doenças da planta. 


\section{Metodologia}

As determinações analíticas para avaliação de parâmetros físico-químicos e do teor percentual dos macronutrientes indicados foram realizadas utilizando procedimentos de referências internacionais e complementados em laboratórios certificados pela ISO/ IEC 17025:2005.

\section{ANÁLISES FÍSICO-QUÍMICAS}

Para a descrição do lodo coletado na indústria (Figura 1), foram realizadas análises físico-químicas no laboratório de química ambiental da Faculdade Tecnologia SENAC de Goiás, além da complementação de outras análises em laboratório credenciado pelo Inmetro. Os parâmetros avaliados foram os seguintes: $\mathrm{pH}$, Umidade, Matéria Orgânica, Resíduo Mineral Fixo, Carbono Orgânico Total, Nitrogênio Total e Fósforo Total.

Os parâmetros de $\mathrm{pH}$, Umidade, Matéria Orgânica e Resíduo Mineral Fixo, foram determinados no laboratório de química da faculdade SENAC e realizadas em triplicata (Figura 2), os parâmetros de Carbono Orgânico Total, Nitrogênio Total e Fósforo Total foram realizados em laboratório credenciado pelo Inmetro. Essas análises levaram em consideração referenciais teóricos e a Resolução do CONAMA n 375 de 2006 (de forma comparativa, já que não se aplica a este tipo de lodo específico) que determina os principais parâmetros a serem analisados no lodo para definir se há ou não, potencial para aplicação agronômica.

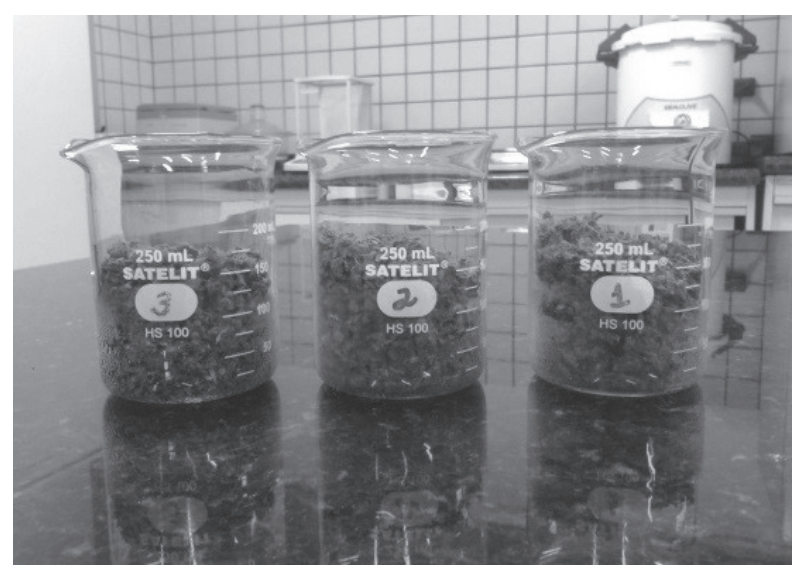

Figura 2. Amostras de lodo coletadas.
pH

Para a determinação de $\mathrm{pH}$, a amostra de lodo foi dissolvida com água destilada (1:10) em um béquer de vidro com o auxílio de um bastão de vidro, seguido da filtração em papel de filtro. O filtrado foi levado para o pHmetro para medição.

\section{UMIDADE}

Foram pesadas 50 gramas da amostra em cápsula de porcelana devidamente identificada. As amostras foram secas a $105^{\circ} \mathrm{C}$ por 2 horas em estufa de secagem. Após o procedimento, as amostras forma resfriadas em um dessecador e posteriormente pesadas em uma balança analítica. Para o cálculo do teor de Umidade, foi utilizadoaa Equação 1.

$$
\%=\frac{100 x\left(p_{c v}-p_{f}\right)}{p_{a}}
$$

Em que:

- $\mathrm{p}_{\mathrm{cv}}=$ peso da cápsula vazia
- $\mathrm{p}_{\mathrm{f}}=$ peso final
-
$\mathrm{p}_{\mathrm{a}}=$ peso da amostra

\section{MATÉRIA ORGÂNICA (MO)}

Para a análise da Matéria Orgânica do método da mufla, foi realizado seguindo o método estabelecido por Goldin (1987), com as seguintes modificações: secagem prévia das amostras em estufa a $105^{\circ} \mathrm{C}$, por um período de $24 \mathrm{~h}$, visando eliminar toda a água presente nos resíduos, como a higroscópica, a capilar ou de cristalização (RODELLA \& ALCARDE, 1994). Após esse período, as cápsulas de porcelana com as amostras foram acondicionadas em forno do tipo mufla e incinerados a uma temperatura de $550^{\circ} \mathrm{C}$, por $3 \mathrm{~h}$. Posteriormente, o conjunto (cápsula + resíduo) foi acondicionado em dessecador e, em seguida, pesado (CARMO \& SILVA, 2012). O teor de matéria orgânica foi determinado em razão da perda de massa do resíduo incinerado, considerando-se o material perdido pela queima no intervalo de variação da temperatura de 105 a $550^{\circ} \mathrm{C}$, conforme a Equação 2 (CARMO \& SILVA, 2012). 


$$
M O(\%)=[P-(T-C) x 100] / P \quad \text { Equação } 2
$$

Em que P é igual ao peso da amostra (g) depois de aquecida a $105^{\circ} \mathrm{C}$; $\mathrm{C}$ é igual ao tara do cadinho (g); $\mathrm{T}$ é igual ao peso da cinza + cadinho (g).

\section{RESÍDUO MINERAL FIXO (RMF)}

Os procedimentos realizados para a análise de Resíduo Mineral Fixo foram determinados de acordo com o Ministério da Agricultura, Pecuária e Abastecimento (MAPA) de 2014. A amostra foi colocada na cápsula de porcelana e deixada na mufla a $550^{\circ} \mathrm{C}$ durante 3 horas, depois deixou esfriar em dessecador e, logo após, a amostra foi pesada em balança analítica, registrando a massa obtida. O Resíduo Mineral Fixo foi calculado através da Equação 3.

$\% R M F=\frac{\left(m_{2}-m_{1}\right) x 100}{m_{0}}$

Equação 3

Em que m2 é igual a massa do cadinho com amostra após incineração, em gramas; m1 é igual a massa do cadinho vazio, em gramas; $\mathrm{m} 0$ é igual a massa da amostra, em gramas.

\section{CARBONO ORGÂNICO TOTAL (COT), NITROGÊNIO TOTAL (NT), FÓSFORO TOTAL (P) E POTÁSSIO (K)}

Para a determinação dos nutrientes do lodo (COT, NT, P e K), foram utilizadas as metodologias de análises ,internacionalmente reconhecidas, baseadas no Standard Methods for the Examination of Water and Wastewater (SMWW, 2012).

\section{Resultados e Discusões}

De acordo com as análises realizadas, para o parâmetro de $\mathrm{pH}$, o valor encontrado para a amostra analisada foi menor que 6. Este valor do $\mathrm{pH}$ tem influência direta do
Policloreto de Alumínio, pois seu pH é baixo, interferindo diretamente no processo gerando uma digestão em meio ácido. Para a determinação da Umidade, encontrou-se um valor estimado de $80 \%$ na amostra de lodo centrifugado. Já para a determinação de Matéria Orgânica, o valor encontrado foi de $92 \%$, em média. Após este procedimento, realizou-se a determinação de Resíduo Mineral Fixo (Cinzas), obtendo-se um valor próximo a $8 \%$. Outras determinações realizadas em laboratório parceiro foram as análises de Carbono Orgânico Total $(36,0 \mathrm{mg} / \mathrm{kg})$, Fósforo total $(2,0 \mathrm{mg} / \mathrm{kg})$, Nitrogênio total $(74,9 \mathrm{mg} / \mathrm{kg})$ e Potássio $(0,8 \mathrm{mg} / \mathrm{kg})$. A tabela 1 apresenta os resultados encontrados para todas as determinações analíticas realizadas.

Baseado nos dados encontrados, observa-se que os resíduos de lodo avaliados têm potencial suficiente para serem utilizados como fertilizantes agrícolas. Os resultados indicam que esse pode ser classificado como fertilizante do tipo misto (combinação de fosfatados, nitrogenados e potássicos). A aplicação do lodo requer uma avaliação prévia tanto do solo a ser aplicado, do manejo e do tipo de cultura. Outros macronutrientes também podem ser avaliados, como cálcio e magnésio.

A Resolução do CONAMA n ${ }^{\circ} 375$ de 2006 considera que o lodo proveniente de esgoto sanitário ou de efluente constitui em sua composição, matéria orgânica e nutrientes favoráveis para as plantas e que sua aplicação no solo traz benefícios tanto para a agricultura, quanto para a silvicultura. Além disso, trata-se de uma alternativa que apresenta vantagens ambientais, quando comparado a outras práticas de destinação final do lodo, considerando também que a aplicação do lodo na agricultura se enquadra nos princípios de reutilização de resíduos de forma ambientalmente adequada, estando de acordo com a Política Nacional de Resíduos Sólidos de 2010. Como não há uma legislação específica à aplicado do lodo de processos industriais, pode-se adotar as práticas estabelecidas no CONAMA n ${ }^{\circ} 375$ como referência técnica.

\section{Conclusão}

Todo o percentual de lodo gerado pela indústria de transformação avaliada (140 toneladas) é encaminhado ao aterro sanitário municipal onde a empresa se encontra. 
O estudo mostra o tamanho do impacto desse resíduo no meio ambiente, além de reduzir drasticamente a vida útil do aterro. Uma boa gestão do resíduo será capaz de dar um destino mais ambientalmente adequado, como o proposto neste artigo, já que foi encontrado altos teores do macronutriente nitrogênio no lodo, indicando uma possível aplicação como fertilizante agrícola (NPK 75$2-0,8)$.

\section{Referências Bibliográficas}

1. Almeida. A. C. et al. Plano de Gerenciamento de Resíduos Sólidos. Instrumento de Responsabilidade Socioambiental na Administração Pública. Brasília, 2014.

2. Carmo, D. L; Silva, C. A. Métodos de quantificação de carbono e matéria orgânica em resíduos orgânicos. Tese (Doutorando em Ciência do Solo) - Universidade Federal de Lavras - UFLA. Revista Brasileira de Ciência do Solo. Viçosa, 2012.

3. Decreto $\mathrm{n}^{\mathrm{o}} 86.955$ de 18 de fevereiro de 1982. Ministério da Agricultura, Pecuária e Abastecimento (MAPA).

4. Fernandes, E.; Dias, V. P. Fertilizantes: Uma Visão Global Sintética. BNDES Setorial, Rio de Janeiro, n. 24, p. 97-138, set. 2006.

5. Goldin, A. Reassessing the use of loss-on-ignition for estimating organic matter content in noncalcareous soils. Commun. Soil Sci. Plant. Anal. 1987.

6. Ministério da Agricultura, Pecuária e Abastecimento - MAPA, 2014. Disponível em: < http://www.agricultura.gov.br/> Acesso em: 25-09-2015.

7. Política Nacional de Resíduos Sólidos, Lei n 12.305 , de 2 de Agosto de 2010.

8. Resolução do CONAMA - Conselho Nacional do Meio Ambiente. N 375 de 29 de Agosto de 2006.

9. Rice; E.W. et al. Standard Methods for the Examination of Water and Wastewater. 22 $2^{\mathrm{a}}$ edição. Pharmabooks Editora. 2012.

10. Rodella, A. A; Alcarde, J.C. Avaliação de materiais orgânicos empregados como fertilizantes. Scientia Agricola. Piracicaba, 1994.

11. Silva, E. C; F. Produção de composto orgânico. Trabalho de Conclusão do Curso de Tecnologia em Cafeicultura (Graduação) Escola Agrotécnica Federal de Muzambinho, Muzambinho, 2008.

12. Valois, R. S. Comissão de Meio Ambiente e Qualidade de vida na Escola (COM-VIDA): análise de uma política pública de educação ambiental e sua implementação em duas escolas de Teresina - Piauí. 2013. Dissertação (mestrado) - Universidade Estadual Paulista Júlio de Mesquita Filho, Instituto de Biociências de Rio Claro, 2013.

\section{William D. Miranda*, Geymme S. C. Soares, Adriano M. Moura \& Vanessa S. Leal.}

Faculdade de Tecnologia SENAC-GO. Av. Independência, 1615, Leste Vila Nova, Goiânia, Goiás, Brasil.

*Email: w.dmiranda@yahoo.com.br. (62) 9680-5844 